\title{
Electrospun ion-conducting composite membrane with buckling-induced anisotropic through-plane conductivity
}

Ariel Odess, ${ }^{1}$ Matan Cohen, ${ }^{2}$ Jian Li, ${ }^{1}$ Mauricio Dantus, ${ }^{3}$ Eyal Zussman, ${ }^{4}$ Viatcheslav

$$
\text { Freger }^{1,2,3 *}
$$

${ }^{I}$ Wolfson Department of Chemical Engineering, Technion - Israel Institute of Technology, Haifa 3200003, Israel

${ }^{2}$ Grand Technion Energy Program, Technion - Israel Institute of Technology, Haifa 3200003, Israel

${ }^{3}$ Russel Berrie Nanotechnology Institute, Technion - Israel Institute of Technology, Haifa 3200003, Israel

${ }^{4}$ Department of Mechanical Engineering, Technion - Israel Institute of Technology, Haifa 3200003, Israel

*E-mail: vfreger@technion.ac.il

\section{S1. Electrospinning setup}

The dual-electrospinning system used in this research is schematically shown in Figure S1. It included an electrically grounded rotating metallic collector positioned between two syringes that feed polymer solutions through metal needles. The needles were held under a $9 \mathrm{kV}$ voltage relative to the collector by means of a power supply; the needles were connected to the respective syringes through a flexible tubing, in order to minimize electrical interferences with the pump electronics. The PVDF and Nafion solutions were 
then fed and resulting fibers were co-deposited on a collector from opposite sides. The collector, needles and tubing were enclosed in a chamber, purged with nitrogen to keep the temperature and humidity within the desired range.

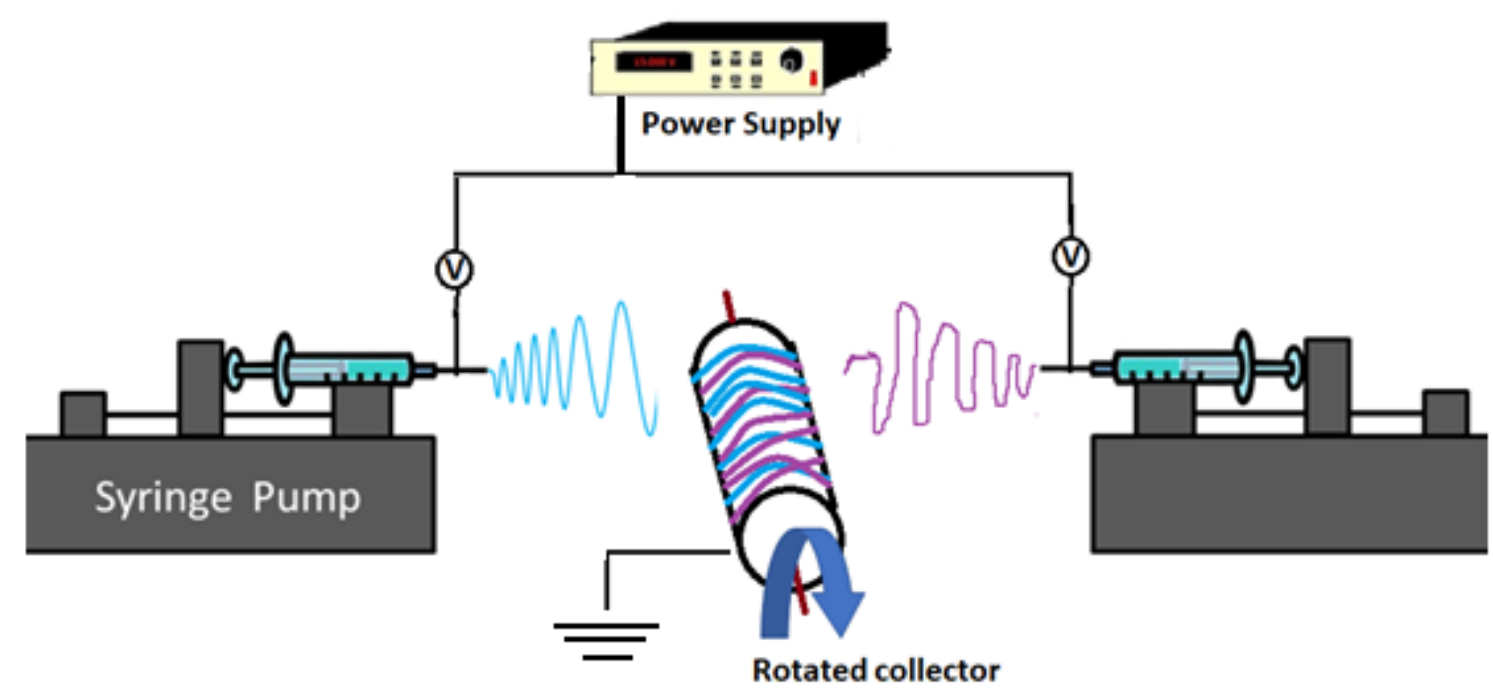

Figure S1. Schematic representation of the dual electrospinning system. Note, in the actual system, the needles were connected to syringes through flexible tubing to minimize interference of the high voltage with the pump electronics.

\section{S2. FTIR analysis of the electrospun mats}

In order to verify uniform mixing of PVDF and Nafion within collected nanofiber mats AFT-FTIR spectra was recorded at several locations across the electrospun mat. Each spot, i.e., the size of the ATR diamond crystal sensor, was $2 \mathrm{~mm}$ in diameter. The resulting spectra are shown in Figure S2. It is seen that the spectra obtained at different locations are virtually identical and are a superposition of the spectra of individual polymers, PVDF and Nafion. 


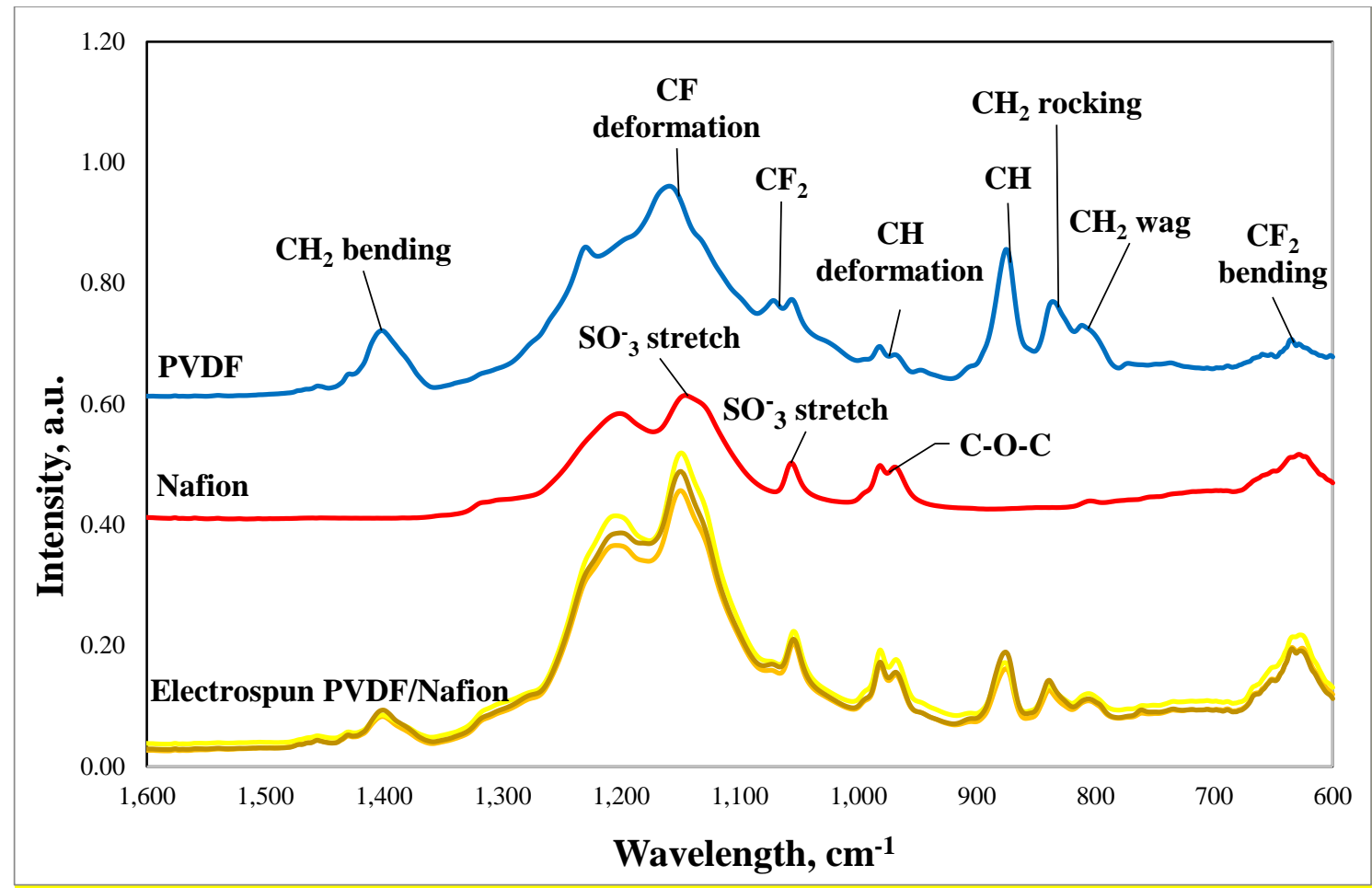

Figure S2. FTIR spectra of cast PVDF (blue), cast Nafion (red) and electrospun PVDF/Nafion membrane. Main peaks and attributed functional groups are labeled.

\section{S3. SAXS: azimuthal dependence of the ionomer peak intensity}

In addition to SAXS 2D maps for B- and P-membranes presented in Figure 4 in the main text, Figure S3 presents azimuth angle dependence of the scattering intensity along the ionomer peak ring. Each point in the spectra is the average over a small azimuth range for a radial q-range ( $q=\sqrt{q_{x}^{2}+q_{y}^{2}}$ ) around $q \sim 0.1 \AA$ - 1 encompassing the entire ionomer ring. The degree of alignment in a direction normal to the incident one is indicated by the amplitude of intensity variation versus azimuth, relative to the average intensity; a larger relative amplitude indicates a stronger ionic channel alignment along the direction defined by the maxima of the intensity. On the other hand, flatter curve corresponds to little alignment in the corresponding direction.

Figure S3 shows that the relative amplitude of intensity variations for a P-membrane is larger, when the incident the beam is normal to the membrane, i.e., perpendicular to the fiber orientation within the P-membrane (panel A), and it is smaller when the incident beam is parallel to the membrane, i.e., goes mainly along the channels (panel C). The 
situation is opposite for a P-membrane (panels B and D), where variations are far larger, when incident beam is parallel to the membrane (panel D) compared to the perpendicular one (Panel B), indicating a well-pronounced TP alignment of ion channels.

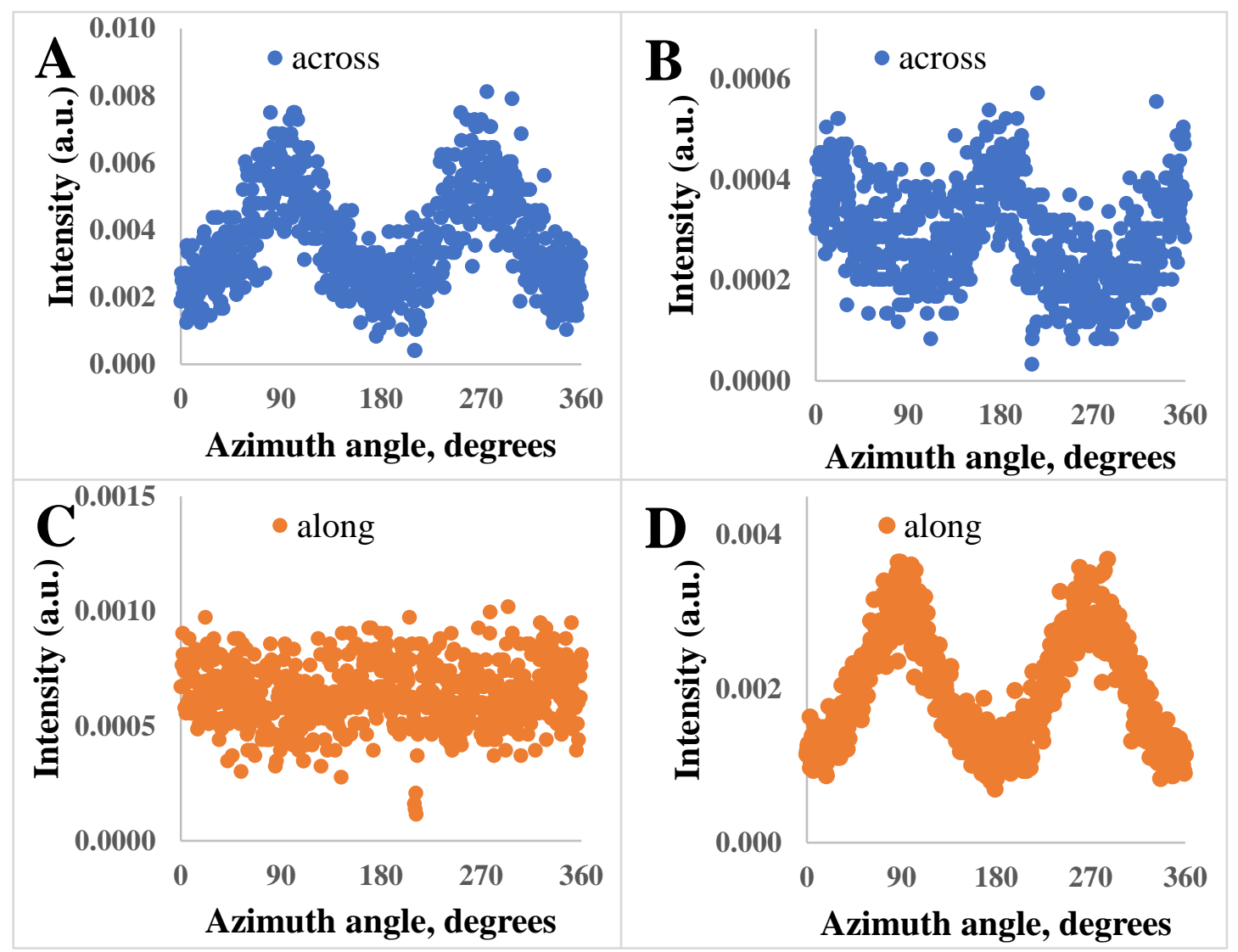

Figure S3. Intensity of the ionomer peak of Nafion in SAXS spectra (at ca. $q \sim 0.1 \AA-1$ ) versus azimuth angle for a P-membrane $(A, C)$ and a $B$-membrane $(B, D)$. The spectra are obtained by averaging the intensity (a.u.) of the ionomer ring in Figure 4 for small azimuth ranges. "Across" $(A, B)$ and "along" $(C, D)$ refer to the direction of the incident beam relative to the original fiber orientation within the as-collected mats.

\section{S4. Impedance measurements}

Figures S4 to S6 illustrate the evaluation of TP conductivity from impedance spectra (blue circles) and its comparison with IP conductivity, indicated by the orange diamond. Accurate evaluation of the latter presents no problem, as it is directly read form the spectra as the value corresponding to the wide plateau of the impedance spectrum in the Bode plot in the range $1 \mathrm{~Hz}$ to $10 \mathrm{kHz}$ or as the intersection with real axis in the Nyquist plot, which 
is well within the measured frequency range and extends over several decades of frequency. However, in the case of TP conductivity, due to the relatively high specific conductivity, large membrane area and small thickness, the corresponding plateau stabilizes at very high frequencies above the upper limit of the frequency range. Therefore the conductivity is evaluated by extrapolating the high-frequency semicircle (left-most part of the spectrum) to the real axis and comparing it with the IP value. For instance, Figure S4 displays the entire spectrum of a Nafion 115 membrane, an isotropic benchmark material, while the inset zooms in to the high-frequency part. It is seen that extrapolation yields a value very close to the indicated IP conductivity, which is confirmed by equivalent circle fitting as the most appropriate way of such extrapolation[1,2]. The close values of IP and TP conductivity indeed indicate Nafion 115 is largely isotropic.

Figures S5 and S6 illustrate the extrapolation for B- and P-membranes, respectively. The blue diamond represents the extrapolated values, obtained by fitting to the equivalent circuit proposed by Cooper ${ }^{1,2}$. For a reliable fitting, unaffected by instrumental artefacts (especially, at frequencies above $1 \mathrm{MHz}$, cf. the leftmost point in Figure S6), the data points for the highest frequencies in the measured spectra were successively removed from the fitted spectra until the extrapolated conductivity converged to a stable value. Regardless of such uncertainties, Figure S5 and S6 clearly show the extrapolated TP value significantly exceeds the IP one for P-membrane (Figure S5) and the opposite holds for B-membrane (Figure S6), indicating a strong anisotropy of both membranes.

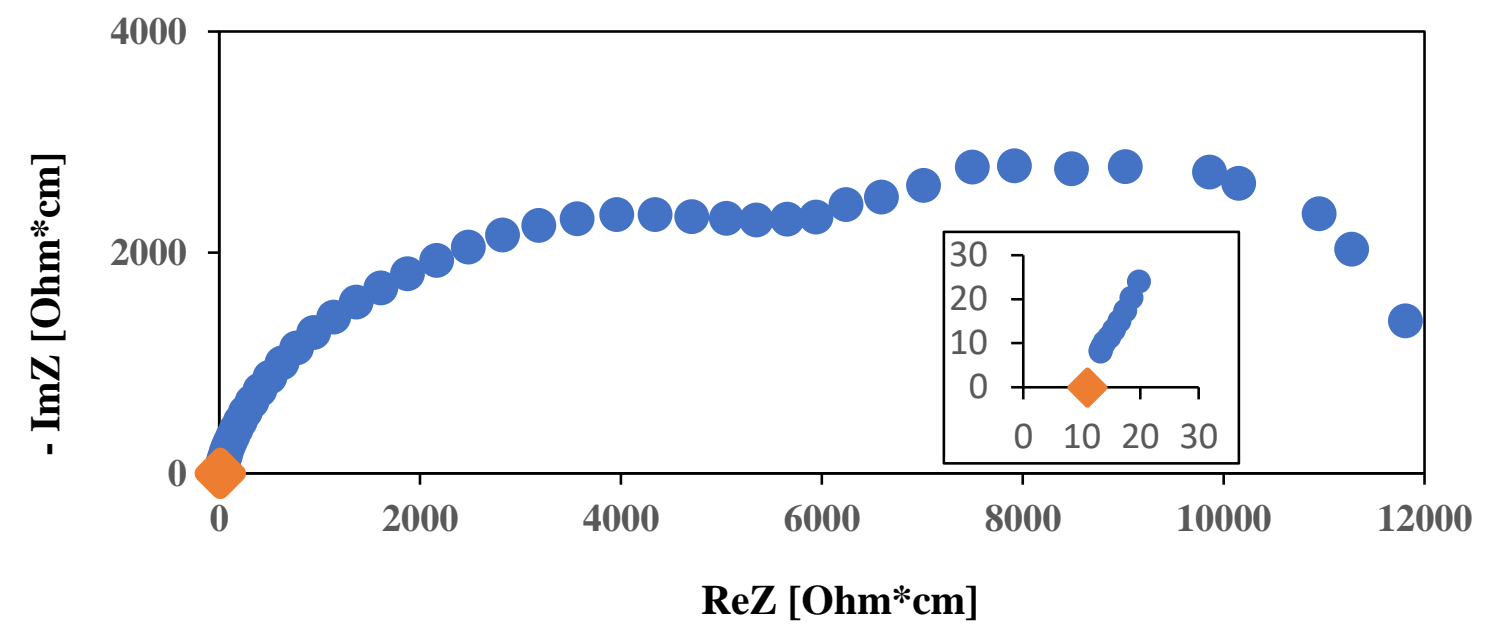

Figure S4. TP impedance spectrum of Nafion 115 (blue circles) compared to its IP resistance (orange diamond). The inset magnifies the high-frequency range. All values are normalized to sample geometry to represent specific resistance values. 


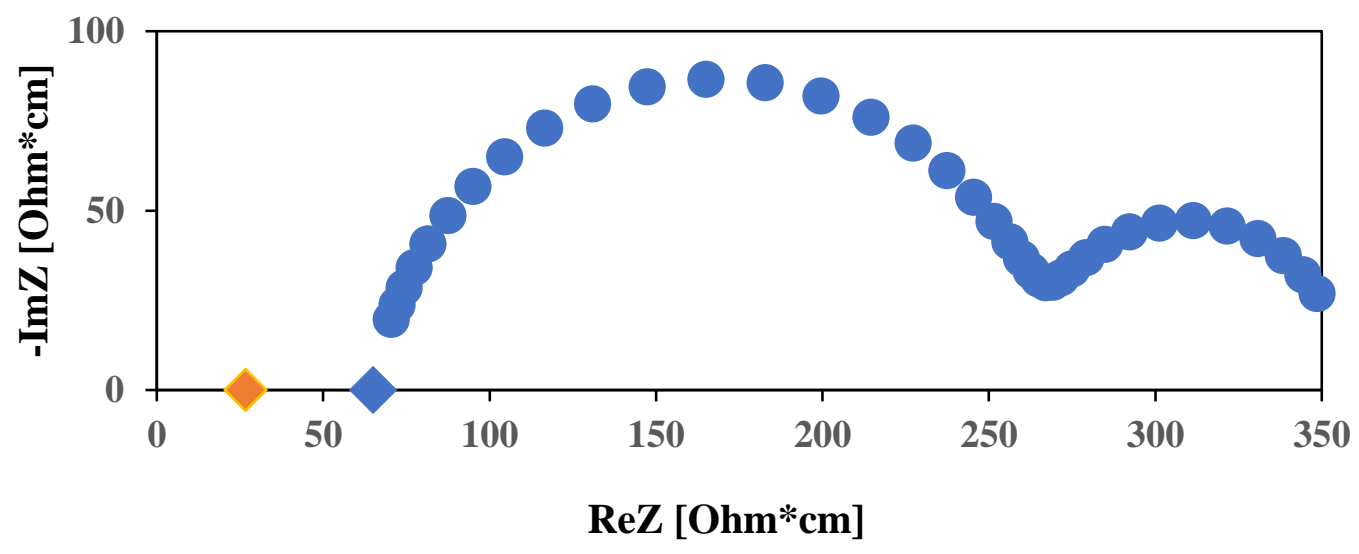

Figure S5. TP impedance spectrum of P-membrane (blue circles) compared to IP resistance (orange diamond). The blue diamond is the extrapolated TP membrane resistance. All values are normalized to sample geometry to represent specific resistance values.

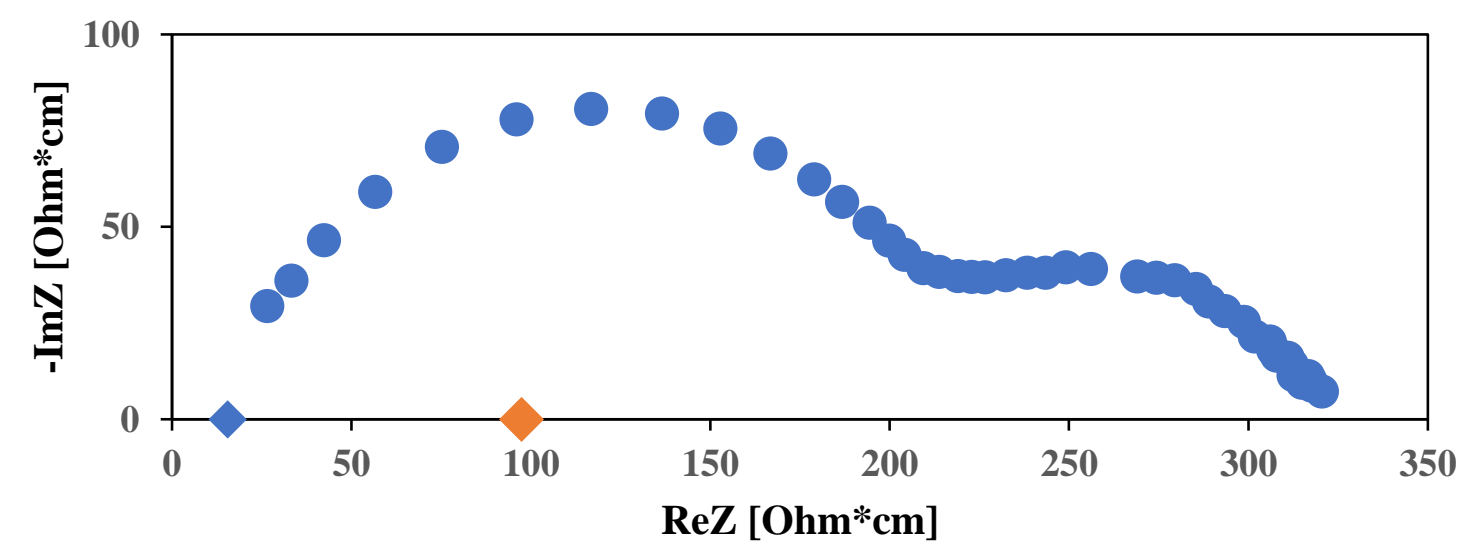

Figure S6. TP impedance spectrum of a B-membrane (blue circles) compared to its measured IP resistance (orange diamond). The blue diamond is the extrapolated TP membrane resistance. All values are normalized to sample geometry to represent specific resistance values. 


\section{S4. Water uptake measurements}

Figures S7 presents an example of water uptake measurements for P- and B-membranes at $30^{\circ} \mathrm{C}$ and stabilization at two relative humidities $(\mathrm{RH}), 90$ and $95 \%$. Each result was considered stabilized until the rate of change dropped below a preset value. The baseline (dry state) was obtained using a flow of dry nitrogen.

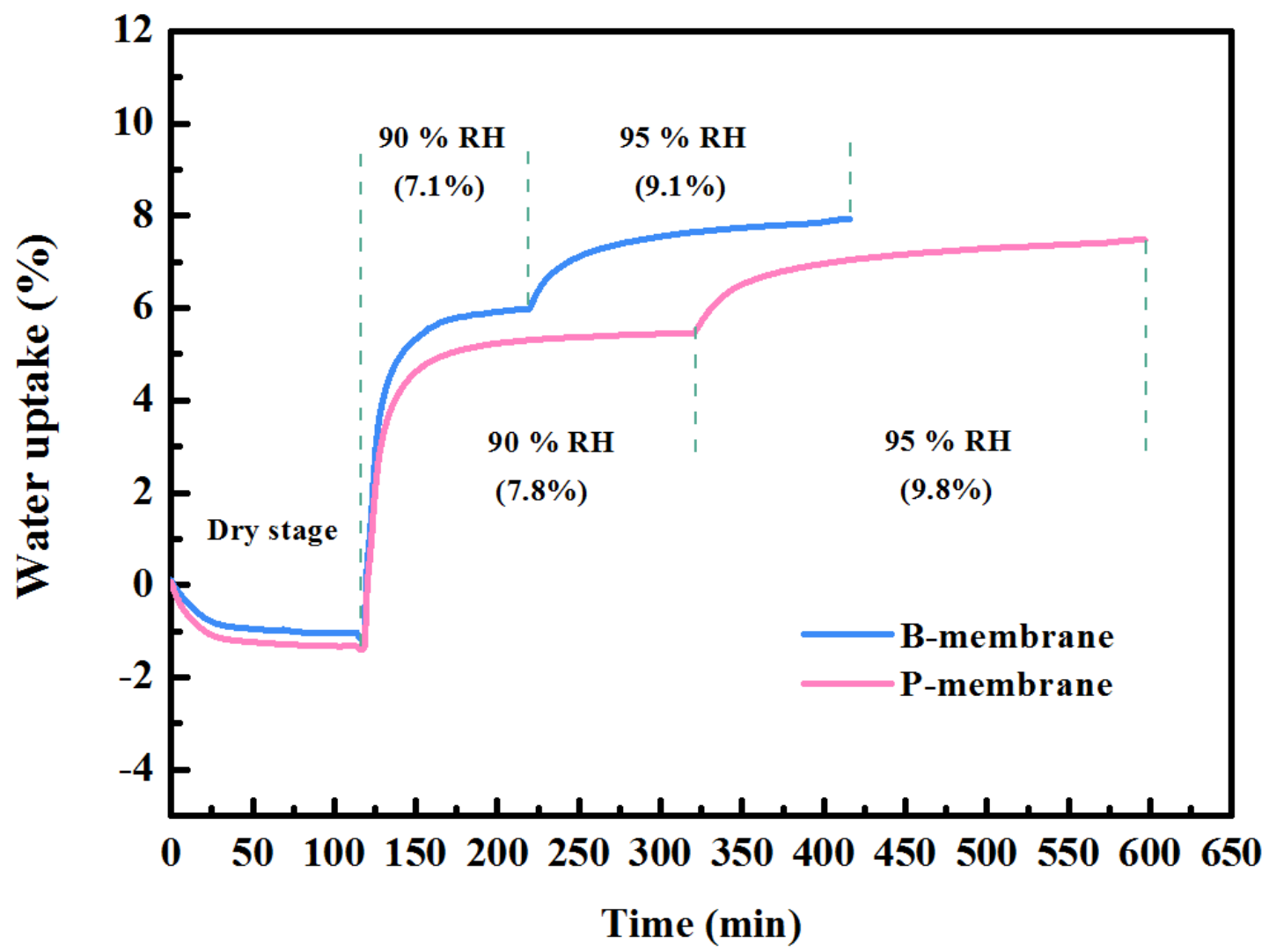

Figure S7. Time-dependent water uptake of the P-membrane and B-membrane at $30^{\circ} \mathrm{C}$.

\section{References}

1. Cooper, K. R. Progress Toward Accurate Through-Plane Ion Transport Resistance Measurement of Thin Solid Electrolytes. Journal of The Electrochemical Society 157, B1731 (2010).

2. Cooper, K. Characterizing Through-Plane and In-Plane Ionic Conductivity of Polymer Electrolyte Membranes. in (2011). 